\title{
Le statut énonciatif des unités dites "transposées" en discours indirect libre
}

\author{
Jean-Daniel Gollut, et Joël Zufferey
}

Section de français, Faculté des Lettres-Anthropole, Université de Lausanne, 1015

Lausanne, Suisse.

jean-daniel.gollut@unil.ch

Résumé. La transposition, concept central de l'approche grammaticale du discours rapporté, a été remise en question par la redéfinition du champ en termes de représentation $d u$ discours autre. Le changement de paradigme a imposé de renoncer au schéma selon lequel un énoncé, censément premier, serait transféré dans un discours d'accueil. Tout en adoptant l'ordre sémiologique de la représentation, les auteurs proposent ici de réhabiliter l'opération de transposition dans la compréhension du discours indirect libre, non sans réévaluer le statut énonciatif des unités concernées. Car si, dans ce cadre, les pronoms de troisième personne, l'imparfait et les temps qui lui sont associés, de même que les localisateurs spatiotemporels sont des formes censées convenir au régime cotextuel de base (plan énonciatif de l'histoire pour le récit classique), elles conservent, en tant que produit d'une transposition, une part de la subjectivité propre au discours représenté. En dépit de leur forme anaphorique, ce ne sont pas de purs morphèmes narratifs amenés à se combiner, dans la syntagmatique du DIL, avec d'autres éléments de nature discursive: ils fonctionnent eux-mêmes à titre de quasi-indicateurs. Cette spécificité des unités transposées par rapport aux domaines d'emploi définis par Benveniste ne va pas sans rendre problématique la conception habituelle du discours indirect libre comme «mélange de voix ».

\footnotetext{
Abstract. The Enunciative Status of Backshifted Units in Free Indirect Speech. A central concept in the grammatical approach of reported speech, backshift has recently been challenged by a new understanding of free indirect discourse as a representation of speech. Such a paradigm shift (from reported to represented speech)
} 
involves abandoning the idea that a first statement is reproduced in a second one. Although agreeing with this general principle, the authors propose here that backshift operations can only explain the very forms of free indirect speech, which imply that the enunciative status of the relevant units is reassessed. Although they seem to work as anaphoric components governed by the narrative context, third person pronouns, the imperfect and other tenses, as well as place and time expressions are the results of backshift operations and maintain the subjectivity of the represented statement. In spite of their anaphoric forms, they are not to be considered as regular narrative morphemes like other discursive components: they operate as quasi-indexicals. This peculiarity of backshifted units, here considered in relation to Benveniste's categories, calls into question the usual comprehension of free indirect speech as a dual-voice phenomenon.

La théorie du "discours rapporté » a longtemps consisté, dans la tradition scolaire comme dans la vulgate grammaticale, à mettre en correspondance systématique deux énoncés selon un ordre orienté de restitution ${ }^{1}$. De là l'idée de transposition, appliquée aux régimes indirects libre et lié, suivant laquelle les désignateurs pronominaux, la temporalité verbale et les indicateurs spatio-temporels d'un discours premier sont convertis lors de leur transfert dans le discours d'accueil. Cette conception binaire d'un énoncé obtenu par transformation d'un discours préexistant a été profondément révisée par [1, 2] J. Authier-Revuz dans le sens d'une sémiologie de l'altérité dans la langue et de ses manifestations en discours: le champ de la représentation $d u$ discours autre a ainsi remplacé, dans les travaux de la linguiste, celui du discours rapporté (cf. 1992-3, 2004). Le changement de paradigme a d'abord évacué la référence systématique à un discours d'origine, objet présumé des diverses restitutions, et a entraîné du même coup la mise en cause de la notion de transposition.

Tout en adoptant l'ordre sémiologique de la représentation, nous emprunterons, de notre côté, une voie en partie différente. Nous pensons en effet pouvoir conserver le concept de transposition en le plaçant même, après l'avoir redéfini, au centre de l'analyse. Ainsi, par ce terme, nous ne supposons aucunement l'idée qu'un discours premier serait, mutatis mutandis, rendu par un second. Suivant une trajectoire inverse, qui impose de réviser profondément le statut des composantes impliquées, le processus cognitif de transposition intervient, selon nous, lorsqu'un énoncé donne à concevoir un autre qui, loin d'amorcer le mouvement de représentation, constitue son 
horizon de compréhension. La transposition concerne alors précisément la configuration de certaines unités langagières que l'énoncé représentant propose de saisir et interpréter à partir de l'image du discours autre qu'il projette.

Du phénomène de la transposition, ce sont moins les principes de régulation formelle que nous voulons examiner ici, que leur incidence sur le fonctionnement énonciatif des unités concernées. De fait, l'acception strictement formelle, appuyée par une règle de «concordance» selon laquelle le verbe du discours reproduit doit s'ajuster quasi mécaniquement au temps du verbe recteur (DI) ou du contexte (DIL), génère des problèmes insolubles ${ }^{2}$. D'autres linguistes ont voulu conserver l'idée de transposition en la redéfinissant sur un plan sémantique (cf. [6] Curat 1991 : 108ss). Ce n'est plus alors une règle de concordance morphosyntaxique, mais le sens, garant d'un domaine de prise en charge, qui organise le jeu des univers temporels. Le verbe du discours représenté n'est plus soumis aux conditions formelles édictées par l'amont textuel; il garde une valeur temporelle propre. Cela permet d'admettre certaines «discordances », par exemple la validation par le présent d'une proposition due à autrui, alors que le contexte d'intégration est au passé. En revanche, ce type d'explication pourra buter sur le cas d'un imparfait, censé garder sa signification pleine de passé, mais qui se trouve pourtant mis en corrélation avec un déictique de postériorité («Il a déclaré qu'il partait demain »).

Il ne s'agit pas ici d'arbitrer entre ces différents partis pris théoriques, mais plutôt de marquer la limite qui leur est commune dans l'analyse des faits de transposition. Car ceux-ci, quelle que soit la part qui leur est accordée dans tel ou tel modèle, restent envisagés de manière partielle. Ainsi, pour ce qui concerne la valeur des tiroirs verbaux, les traits pris en considération relèvent invariablement et uniquement des plans temporel et aspectuel. Compte tenu cependant des déplacements d'ancrage inhérents aux représentations indirecte et indirecte libre, la question du statut énonciatif des unités qui se donnent comme transposées mérite bien d'être également soulevée. Faut-il admettre que les temps du «passé » (plus-que-parfait, imparfait, conditionnel) qui interviennent, en signifiant leur transposition, dans un contexte énonciatif historique soient de facto assimilés, dans leurs formes et leurs valeurs, au régime narratif de base ? Voilà ce que nous entendons discuter, mais pas seulement à propos de la morphologie verbale. Car c'est l'ensemble des unités interprétables dans une logique de transposition- personnes, temps verbaux, indicateurs spatio-temporels qui se trouve impliqué dans la problématique énonciative. Un pronom de troisième personne qui se lit comme transposant un JE endosse-t-il pleinement le statut de non-personne? Un morphème conditionnel, qui 
prétend transcrire un futur, signifie-t-il une relation purement objective d'ultériorité temporelle? Et de même encore pour les anaphoriques transpositeurs (demain > le lendemain...), dont on peut se demander s'ils naturalisent complètement l'indicateur dans le système du texte rapporteur. Ces questions, par principe applicables à tous les types de discours indirect, nous allons ici les traiter, avec quelques spécificités, dans le cadre du discours indirect libre. On verra que la notion de transposition, affranchie d'une définition strictement grammaticale, reste un fondement pour l'interprétation du DIL.

Les exemples qui serviront à la démonstration seront tous prélevés de récits exprimés au régime historique. Ce dispositif narratif présente un double intérêt : d'une part, il correspond à la conception la plus consensuelle du DIL et, d'autre part, il permet d'interroger frontalement la manifestation formelle dans le DIL de deux voix, dualité soutenue tant par [7] V. Volochinov (1929) que par [8] R. Pascal (1977). Il serait cependant trop restrictif d'exclure catégoriquement de notre domaine d'investigation les cas pouvant apparaître dans des énoncés à la première personne. Sur ce point, nous préciserons nos vues en conclusion.

\section{La subjectivation de IL : de l'anaphorique au quasi- indexical}

Par l'opération présumée de transposition, le pronom représentant le locuteur dans le discours indirect libre se donne à concevoir comme la forme correspondant à une énonciation personnelle: le sujet désigné dans le discours représenté par un embrayeur (JE) se retrouve dans le DIL - au terme d'un recalcul référentiel basé sur un nouvel ancrage - désigné par rapport à l'instance de parole en activité. En régime de narration hétérodiégétique, la transposition s'impose à l'interprétation lorsque le IL (ainsi que ses déclinaisons grammaticales elle, le, la, lui, et les possessifs de troisième rang) semble tenir lieu d'un JE. Cette valeur est alors signifiée par l'association à la troisième personne de marques de subjectivité en principe inhérentes à un discours embrayé sur l'activité d'un locuteur (JE), comme on peut l'observer dans les illustrations suivantes :

Comme le bal déjà lui [Emma] semblait loin ! Qui donc écartait, à tant de distance, le matin d'avant-hier et le soir d'aujourd'hui ? (Flaubert, Madame Bovary, 1857 ; nous soulignons)

Il [Pierre] le [le docteur Chassaigne] regardait, stupéfait de ce qu'il lui entendait dire de la Grotte et de Bernadette. Lui, une tête si solide, un 
savant d'une intelligence si exacte, dont $i l$ avait tant admiré autrefois les puissantes facultés d'analyse ! (Zola, Lourdes, 1894)

Les indicateurs de temps (avant-hier, aujourd'hui, autrefois), les types de phrases (interrogation, exclamation), la syntaxe nominale (lui, une tête si solide, un savant d'une intelligence si exacte), les intensifs (comme, tant, si) et autres jugements évaluatifs (déjà, loin, solide, exacte, puissante), ainsi que la thématisation connotant ici l'étonnement (lui, une tête...) sont autant d'éléments ou de traits de discours qui composent l'expressivité subjective $\mathrm{du}$ personnage désigné par les pronoms de troisième personne lui/il (soulignés par nous dans les deux extraits).

Cependant, comme on le voit en particulier dans le second exemple, le DIL peut comprendre à la fois des pronoms de troisième rang transposés et d'autres qui ne se sont pas. Quoique homonymes lorsqu'elles occupent des fonctions syntaxiques similaires, les diverses troisièmes personnes possèdent des statuts énonciatifs foncièrement différents. Seuls les IL (et variantes) transposant un JE sont les représentants d'un SOI et peuvent endosser la subjectivité présente dans l'énoncé. En l'absence de distinction formelle des désignations, et sans possibilité de "remonter» à un authentique discours premier pour y vérifier la distribution des embrayeurs, on peut se demander si les pronoms de troisième personne en cause se différencient in situ par leur fonctionnement référentiel. Car le IL transposant un JE pourrait conserver quelque chose du mode d'occurrence de la première personne en ne se laissant pas réduire à la simple reprise d'une expression référentielle antérieure. Autrement dit, en termes d'analyse de la cohésion textuelle, il s'agit de savoir si les différents IL de l'énoncé, comme le voudrait normalement le statut en langue du pronom, et à l'instar de ceux qui réfèrent à des entités objectives, sont tous de véritables anaphoriques ${ }^{3}$.

Si la question est franche, la réponse n'est pas simple et encore moins l'administration de la preuve. Car l'analyse est tributaire de la complexité attachée à la définition et au fonctionnement de l'anaphore. Si l'on opte pour une conception stricte, l'anaphore est la reprise d'un antécédent textuel. Néanmoins, la mise en relation des termes repose sur une interprétation. Dans le cas de l'anaphore pronominale, la recherche de l'antécédent peut certes être guidée morphologiquement par le genre grammatical (masculin ou féminin) du substitut; mais si plusieurs candidats restent en lice, il faut d'autres critères pour sélectionner l'antécédent adéquat. La macro-syntaxe de l'anaphore doit alors s'articuler avec une théorie cognitive de l'accessibilité référentielle. C'est en fonction de son caractère «saillant » que l'antécédent adéquat peut se faire reconnaître. Reste à définir ce qui produit cette saillance, et ici les considérations sont multiples et hétérogènes : l'accessibilité peut 
dépendre simplement de la proximité textuelle des termes concernés, mais répond aussi à toutes sortes de mises en évidence sémantiques et syntaxiques de l'antécédent (rôle actanciel, statut topical, fonction de sujet, etc.). Si l'on ajoute que le calcul référentiel procède volontiers par voie d'inférence, on comprend que l'existence ou l'absence de la dépendance anaphorique s'avère parfois difficile à démontrer.

Le fait est que le sujet de conscience se trouve d'habitude bel et bien désigné par une expression nominale dans les parages de l'énoncé contenant le pronom. Le DIL est normalement préparé par la mise en place d'une situation d'énonciation (au moins potentielle), laquelle suppose la nomination d'un personnage susceptible de tenir un discours. Mais cela ne dit pas encore si le sujet pronominal du DIL est désigné relativement à cette donnée cotextuelle. Car le passage d'un régime énonciatif à un autre (du pur récit au DIL) est de nature à refonder les paramètres du système référentiel. Cela produit une transformation de la chaîne anaphorique en relation de coréférence, où les expressions désignatives employées de part et d'autre ne sont pas reliées entre elles par un dispositif formalisé de reprise linéaire, mais par le renvoi autonome à un même objet. Ainsi, la récurrence formelle du pronom peut-elle recouvrir des réalités de fonctionnement fort différentes, eu égard au statut énonciatif des emplois considérés :

La vraie vérité était que Lantier, très bavard sur les autres, se taisait ou mentait quand il s'agissait de lui. Il ne voulait même pas dire où il demeurait. Non, il logeait chez un ami, là-bas, au diable, le temps de trouver une belle situation. (Zola, L'Assommoir, 1877)

Dans la succession des pronoms désignant Lantier, trois sont anaphoriquement des maillons de la chaîne référentielle initiée par le nom propre (il s'agissait de lui, il ne voulait, il demeurait); en revanche le segment non, il logeait chez un ami, là-bas, au diable, le temps de trouver une belle situation, en tant que DIL, comprend une troisième personne subjectivée dont la référence est directement assurée, à l'instar de celle d'un embrayeur, par l'exercice même du discours représenté.

Cette analyse de l'inscription du SOI dans le DIL peut d'ailleurs trouver une confirmation au niveau syntaxique, puisqu'il est possible de trouver en incise attributive, après le pronom, l'expression qui désigne nommément le sujet : «It was a great mistake to have come. He should have stayed at home and read his book, thought Peter Walsh»(Woolf, Mrs Dalloway, 1925) ${ }^{4}$. Une telle construction, qui déroge à l'ordre nécessaire d'une relation anaphorique, n'est pas celle qui convient à un pronom de « reprise »; elle est plutôt analogue à celle qui s'applique aux énoncés embrayés, où la première 
personne du locuteur et le nom compris dans le segment attributif sont en relation indirecte de coréférence, mais pas d'anaphore. Cette parenté de fonctionnement entre le discours rapporté direct et le DIL porte à penser que le IL sujet du DIL, malgré son statut grammatical de troisième personne, revêt certaines propriétés énonciatives attachées à l'embrayeur JE. De sorte que, sans avoir à tabler sur l'appui nécessaire d'un antécédent, le processus d'identification du IL subjectivé serait l'application d'une instruction pragmatique du type : assigner au pronom la référence à l'individu faisant office d'énonciateur.

Ces considérations s'inscrivent, nous l'avons dit, dans une conception de l'anaphore comme simple relais. Mais une théorie approfondie de l'anaphore pronominale peut également conduire à y soustraire le cas du sujet du DIL. En effet, plus qu'une simple relation de terme à terme entre une expression substitutive et un antécédent, la reprise anaphorique par le pronom de troisième personne doit en principe impliquer la conservation des données sémantiques attachées préalablement à la conception de l'objet représenté. Reprendre anaphoriquement par un IL revient à prolonger implicitement l'état des connaissances acquises sur l'entité désignée ; IL, c'est censément le même :

Il ne renvoie pas directement au texte, mais met en jeu la mémoire immédiate des locuteurs. Même en situation de référence « textuelle», il n'y a pas de remontée à l'antécédent, mais renvoi à la représentation mentale du référent telle qu'elle s'établit au moment de l'énonciation du pronom. ([10] Kleiber $1994: 67)$

Or le IL subjectivé du DIL, en tant que source d'une énonciation distincte de la narration, n'est pas pris dans une contrainte de cohérence par rapport à ce qui est dit de lui dans les énoncés antérieurs. Son image est celle qui résulte de son point de vue singulier, lequel n'est pas forcément conforme à la vision auctoriale exercée par ailleurs (on parle de «DIL dissonant ») :

Coupeau ne connaissait qu'un remède, se coller sa chopine de cric, un coup de bâton dans l'estomac, qui le mettait debout. Tous les matins, il guérissait ainsi sa pituite. La mémoire avait filé depuis longtemps, son crâne était vide ; et il ne se trouvait pas plus tôt sur les pieds, qu'il blaguait la maladie. Il n'avait jamais été malade. (Zola, L'Assommoir, 1877)

Compte tenu des états de faits dûment décrits dans les pages qui précèdent (multiples crises de delirium causées par un alcoolisme chronique), la 
dernière phrase de l'extrait contient une contre-vérité manifeste; ce ne peut être une assertion de l'instance narrative. L'interprétation correcte consiste donc à attribuer la responsabilité énonciative à Coupeau lui-même et à tenir le sujet IL personnellement comptable de l'absurde déni.

Ces quelques observations et réflexions sur le mode de fonctionnement du pronom représentant l'énonciateur du DIL peuvent trouver un étayage théorique dans la définition des quasi-indexicaux. Le concept provient de la philosophie analytique ([11] Perry 1993), où il sert à déterminer le domaine de validité de certaines unités du discours rapporté, et a été repris sur le terrain plus spécifiquement linguistique ([12] Reboul 2000) avec une application particulière au DIL.

L'analyse de Perry met en évidence le fait que, pour maintenir l'efficacité causale d'un énoncé, il faut le laisser ancré dans son système d'embrayage, à savoir dans l'expression du sujet en première personne; JE fonctionne alors comme un indexical essentiel. «Je mets du sucre partout» : cet énoncé, du fait de son embrayage, est de nature à déterminer logiquement ma réaction (remédier au problème). Envisageons maintenant ce qui se passe dans le discours rapporté :

Supposons que quelqu'un veuille rapporter le discours de Perry [Je mets du sucre partout] sans lui ôter son efficacité causale. Pour ce faire, on pourrait considérer que sa seule possibilité est : John Perry a pensé : "Je mets du sucre partout », puisque c'est la seule phrase dans laquelle le pronom de première personne est conservé, alors que John Perry a pensé qu'il mettait du sucre partout ou Il mettait du sucre partout, pensait John Perry, utilisent la troisième personne. Mais est-ce bien le cas? Et ne faudrait-il pas faire de différence entre deux types de pronoms de troisième personne dans le style indirect libre, ceux qui sont utilisés pour faire référence à des objets ou à des individus différents du sujet de conscience et ceux qui sont utilisés pour faire référence au sujet de conscience? (Reboul 2000 : 12)

Il ne peut être question de rendre compte en détail de l'analyse très substantielle d'Anne Reboul. On retiendra seulement le résultat du propos, selon lequel les pronoms désignant le sujet de conscience dans le DIL sont à considérer comme des quasi-indexicaux essentiels. Cela résulte du fait que « l'attribution de référents à ces pronoms est extrêmement semblable, si ce n'est identique, à l'attribution d'un référent au pronom de la première personne », le processus d'attribution ne passant pas par la recherche d'un antécédent, mais s'appuyant globalement sur la description de la situation d'énonciation pertinente (ibid., 23-24). Et c'est en définitive parce que les 
quasi-indexicaux qui y apparaissent sont essentiels que les représentations des états mentaux au DIL pourront exercer une influence sur les décisions à suivre - avoir un poids causal - et donner une expression privilégiée de la subjectivité d'autrui (ibid., 28).

\section{La valeur subjective des temps verbaux transposés}

De même que le pronom IL désignateur du SOI dans le DIL se distingue foncièrement, sur le plan énonciatif, de toute «troisième personne » objet du récit, les temps verbaux transposés ne sont pas équivalents à ceux qui apparaissent en régime proprement narratif. Quoique les formes utilisées de part et d'autre soient pour la plupart les mêmes, les IMP, PqP et $\mathrm{COND}^{5} \mathrm{du}$ DIL possèdent une teneur subjective que n'ont pas leurs homonymes de l'énoncé historique. Cette valeur est rendue possible par certaines propriétés des morphèmes fondées en langue, mais se trouve actualisée dans l'interprétation par transposition, et cela quelle que soit la perspective temporelle installée.

La signification s'appréhende facilement dans les emplois du COND. En régime purement narratif, hors emploi modal, le COND donne une indication essentiellement temporelle de postériorité :

Par des compliments aussi bien placés que ses cadeaux, Bonaparte désarma celui qui deviendrait bientôt son rival et son adversaire. (Bainville, Le dix-huit brumaire et autres écrits sur Napoléon, in [13] Popescu 2013 : 17)

Avec le COND, l'historien évoque ici ce qui adviendra plus tard, mais à titre de réalité aussi sûre et objective que ce qu'il narre au PS. Il anticipe simplement, par une " prolepse », sur le cours des événements. Par contre, le COND inclus dans un DIL ne décrit pas tant un fait postérieur qu'une projection mentale, une appréhension spéculative de l'avenir ; c'est ce qu'un sujet de conscience envisage du futur :

Avant de partir en vacances, il [Frédéric] eut l'idée d'un pique-nique, pour clore les réunions du samedi.

Il s'y montra gai. Mme Arnoux était maintenant près de sa mère, à Chartres. Mais il la retrouverait bientôt, et finirait par être son amant. (Flaubert, L'Éducation sentimentale, 1869)

Dans l'idée de justifier l'interprétation des deux dernières phrases comme relevant d'un DIL et non d'une narration objective, les commentateurs ${ }^{6} \mathrm{n}$ 'ont 
pas manqué de montrer l'incompatibilité de ces énoncés avec ce qui est vérifié dans la suite du récit. Mais la différence ne se situe pas seulement sur le plan ontologique, c'est aussi une affaire de linguistique énonciative : ce n'est pas ici le narrateur-auteur qui nous instruit de ce qui va advenir, mais le personnage de Frédéric qui imagine un futur selon ses désirs. Les COND retrouverait et finirait, en tant que signifiant un FUT par transposition, gardent une part de la vision subjective inhérente à ce temps verbal. Que ce type d'énoncé ait le sens d'une promesse ou d'une conviction, il reste néanmoins tributaire du sujet qui le produit.

La valeur de perspective subjective véhiculée par le COND transpositeur s'étend dans le DIL à la tournure allait + infinitif avec laquelle on le voit facilement alterner :

La faillite !... Elle s'assit, épouvantée, anéantie. Depuis quelques heures, la ruine de son bonheur lui avait fait oublier celle de la maison ; mais elle se souvenait maintenant. Ainsi son mari était ruiné. Tout à l'heure, en rentrant, il allait apprendre son désastre, et il apprendrait en même temps que sa femme et son enfant venaient de partir. (Daudet, Fromont jeune et Risler aîné, 1874)

Cet emploi, qui s'entend ici comme la transposition d'un FUT périphrastique, s'oppose - comme pour les deux COND homonymes - à celui qui, dans une narration historique, aurait valeur d'information anticipée ( « il allait, devait, perdre la vie dans son voyage »).

La vision subjective, sensible dans l'emploi du COND transpositeur, se laisse-t-elle percevoir aussi bien dans les autres temps verbaux du DIL ? La forme temporellement symétrique du COND, le PqP, est-elle investie elle aussi par le point de vue du SOI ? On pourrait répondre que, dès lors que l'énonciation du DIL est rapportée à un IL sujet de conscience, tous les prédicats seront placés sous cette même dépendance. Mais vérifions tout de même l'implication subjective en jugeant sur pièce :

Elle [Emma] s'étonnait, à présent, de n'avoir pas songé à lui [Léon] tout d'abord; hier, il avait donné sa parole, il n'y manquerait pas. (Flaubert, Madame Bovary, 1857)

Si l'énoncé au COND (il n'y manquerait pas) est évidemment pris en charge par le personnage d'Emma, celui au PqP (il avait donné sa parole) qui lui sert d'argument n'en est pas dissociable. C'est bien dans l'esprit d'Emma que le fait passé est appelé à justifier la vision d'avenir. La corrélation du $\mathrm{PqP}$ avec le déictique hier confirme ce point de vue. À la 
différence de ce qui est signifié par le COND, le procès au PqP n'entre cependant pas en contradiction avec la vérité du récit. De même, la forme d'antériorité est en adéquation avec le système temporel du discours narratif. Mais le $\mathrm{PqP}$ n'est pas pour autant assimilable à une forme objective d'antériorité dans le système historique : par son inclusion dans le DIL, il reste le signifiant d'une rétrospection, équivalent d'un PC relatif à l'actualité $\mathrm{du}$ personnage. On peut encore constater ce statut subjectif du PqP dans l'extrait suivant, du fait des structures interrogatives qui l'incluent :

Au petit jour, il [Graffeteau] se réveilla. Il regarda son uniforme, la poussière et la boue de ses brodequins. Où avait-il été, la veille ? Qu'avait-il fait?... Il ouvrit sa fenêtre. Il vit les palmiers et les mimosas du jardin, verts et frissonnants sous la rosée, l'ondoiement de la cime des pins sous le ciel. (Bourget, Lazarine, 1917)

Il n'est guère envisageable que les interrogations soient celles d'une voix narrative ignorante des événements antérieurs de l'histoire; le questionnement sur le passé est bien le fait du personnage. Quoique l'indicateur temporel (la veille) soit dans ce cas de type anaphorique, à l'instar de ceux qui peuvent fonctionner dans un récit pur, le couple qu'il forme dans le DIL avec le PqP n'indique pas tant un retour en arrière dans la chronologie de l'histoire qu'une tentative de recouvrement mémoriel de la part du sujet.

Le principe de subjectivation s'applique finalement aussi à la forme dévolue à l'expression de la simultanéité temporelle : l'imparfait. Depuis que la linguistique s'intéresse au DIL ([14] Tobler 1887), rien n'a été davantage souligné que l'affinité de cette forme de discours avec l'IMP du français. Quoique ce temps verbal ne soit pas propre au DIL, il y a en effet quelque motif de lui reconnaître un caractère emblématique. Cependant, Charles Bally avait très tôt remarqué un risque de confusion :

Ainsi l'imparfait était dans: Il disait qu'il était malade est complètement différent du même imparfait dans : Il était malade et ne put venir. [...] C'est un cas d'homonymie syntaxique. Vouloir expliquer les temps du style indirect libre comme des temps non transposés me semble reposer sur la méconnaissance de la notion de valeur en linguistique statique. ([15] Bally $1914: 419)$

La spécificité de l'IMP en DIL procède de l'exclusivité du morphème, dans la tranche temporelle qu'une narration historique peut occuper pour sa part au moyen du binôme IMP / $\mathrm{PS}^{7}$. Et le monopole de l'IMP n'est pas une 
affaire de subordination grammaticale, puisque le DIL n'est pas dépendant d'un verbe régisseur. Il s'agit plutôt d'une dépendance sémantique, inhérente au statut énonciatif du DIL. Quand il fonctionne en narration historique, l'IMP tient son assise temporelle du PS avec lequel il est associé. En l'absence de temps pivot, c'est un autre repère qui doit être trouvé. On touche là au principe structural qui fonde la subjectivation de l'IMP : le DIL naît précisément du fait que l'IMP se voit donner pour point d'ancrage implicite l'acte d'énonciation attribuable à un sujet de conscience. Dès lors, le temps désigné par l'IMP se conçoit comme le présent de ce sujet. L'aspect sécant de l'IMP est d'ailleurs propice à la focalisation interne et par là-même au rendu de la situation d'expérience d'un $\mathrm{SOI}^{8}$. Dans la ligne de réflexion que nous suivons ici, on soulignera la correspondance qui existe entre le PR et l'IMP sur le plan aspectuel, ce qui permet au second de fonctionner comme équivalent transposant le premier.

Ce rôle transpositeur, rien ne le fait mieux apparaître que, dans l'exemple suivant, l'application de l'IMP à une expression foncièrement liée à l'activité discursive et à la phraséologie :

Il [Raymond] baissa la tête. Une complicité dévouée à ce religieux rêve ? Obéir? ... Continuer de les servir sans tenir compte de la mort ? - Quelle étrange idée ! ... Tiendrait-elle une nuit ? ... Demain, demain, hélas ! ... ah ! Qui savait ? ... Peut-être !... - Projet sacré, après tout ! — De quel droit réfléchissait-il ?...

Il sortit de la chambre, exécuta les ordres à la lettre. (Villiers de l'IsleAdam, « Véra », Contes cruels, 1874 ; italique dans le texte)

Ce sont ici les réflexions du serviteur du comte d'Athol, qui sont rendues au DIL: Raymond, constatant que son maître vit dans l'illusion de la survivance de son épouse, se demande s'il doit se faire le complice de cette folle croyance. Dans le flot tumultueux des questions et des exclamations, Qui savait? est une tournure qui est un pur décalque de son emploi naturel au présent (Qui sait ?). Par différence avec la visée référentielle que pourrait avoir l'énoncé à l'IMP s'il était pris en charge par un narrateur, Qui savait? n'est pas une véritable interrogation sur l'identité d'un agent; le sens reste exactement celui de la locution adverbiale qui, dans le discours représenté, a pour seule fonction de modaliser épistémiquement l'énonciation du sujetpersonnage.

Terminons cette partie sur les temps verbaux en réglant le cas d'un certain emploi de l'IMP dans le DIL, assez banal en fait, mais qui pourrait apparaître contraire à notre thèse de la transposition. [3] Berthonneau \& 
Kleiber (1997 : 129-130), dans une critique de la « concordance des temps », disent que l'IMP [de DI] ne peut pas être vu simplement comme une forme qui transpose un PR du discours premier, puisque l'IMP peut déjà y figurer : il semblerait donc que, dans ce cas, l'IMP ne soit pas analysable comme une forme transposée dans le système temporel du verbe principal ${ }^{9}$.

Nous n'avons rien à défendre en ce qui concerne la concordance; mais la question de la transposition est plus sérieuse. On trouve en effet, dans le DIL comme dans le DI, des IMP qui semblent reprendre purement et simplement ceux qui sont censés figurer déjà dans le discours premier :

De nouveau, tout le passé, à Doinville, défila, l'enfance, la jeunesse. Était-ce au fond des massifs du grand parc ? était-ce dans le détour perdu de quelque corridor du château? Déjà le président songeait donc à elle, lorsqu'il l'avait gardée, à la mort de son jardinier, et fait élever avec sa fille ? (Zola, La Bête humaine, 1890)

Le travail de remémoration, par lequel Séverine cherche à reconstituer l'histoire trouble de ses relations avec le président Grandmorin, figure une énonciation censée user elle-même de l'IMP. Est-ce dire que le DIL ne fait qu'emprunter le temps verbal de ce discours présumé ? Ce serait se laisser prendre à une apparence toute formelle et ignorer le fait que le système des tiroirs du passé accorde à l'IMP deux positions temporelles distinctes : l'une est la coïncidence avec le moment repère ; l'autre est alignée sur un point d'incidence donné par un adverbe d'antériorité ou par un PqP (cf. avait gardée, dans l'exemple précédent). Il n'y a pas normalement d'ambiguïté entre ces deux emplois :

Il était loin Olivier! Il était là-bas, dans la masse de pierre de ce Paris ! Autrefois il était ici chez lui, dans ce logis aimé ! (Claretie, Le Million, 1882)

Dire que, la veille au soir, chez les Boche, on l'accusait d'exagérer le tableau! Ah bien! Elle n'en avait pas fait la moitié assez ! Maintenant, elle voyait mieux comment Coupeau s'y prenait. (Zola, L'Assommoir, 1877)

Dans l'extrait de Claretie, les deux premiers IMP (était loin; était là-bas) renvoient à l'actualité du sujet de conscience, tandis que le troisième, fixé par autrefois, traduit une perspective d'antériorité. Dans l'exemple de L'Assommoir, c'est une succession inverse entre un IMP d'antériorité (la veille... on l'accusait) et un IMP d'actualité (maintenant elle voyait). En 
dépit d'un recours à des unités morphologiquement identiques, homonymie imposée par l'économie du système linguistique, le DIL exploite bien deux valeurs énonciatives distinctes de l'IMP : tantôt un IMP transpositeur du PR ; tantôt, par un décalage dans le système temporel, un IMP fonctionnellement transpositeur d'IMP.

\section{Le repérage des localisateurs spatio-temporels}

Pour mener la démonstration à son terme et montrer que les expressions qui se comprennent par transposition ne donnent jamais lieu, en DIL, à un repérage référentiel de type anaphorique, il reste encore à examiner le cas des localisateurs. Il nous faut donc expliquer, conformément à notre thèse générale, en quoi les localisateurs procèdent à la façon des embrayeurs, alors même qu'ils paraissent adaptés aux conventions du dispositif historique ${ }^{10}$. Il serait à vrai dire plutôt étonnant que ce type d'indicateurs se singularise par un fonctionnement énonciatif irrégulier au regard des autres unités transposées. De fait, le phénomène se présente, en ce qui les concerne, de manière différente de ce que nous avons pu observer pour les domaines de la personne et des temps verbaux. Dans ces deux derniers cas, le système des formes donne à voir les raisons pour lesquelles les occurrences transposées résistent à se fondre intégralement dans le dispositif énonciatif de l'histoire : la subjectivité exprimée dans l'énoncé ne trouve en effet qu'une troisième personne comme support ; quant aux temps verbaux inscrits dans le passé, ils sont privés du repère, le PS, qui permet de les situer dans l'ordre d'une temporalité objective. Rien de tel cependant pour les localisateurs transposés, qui ne sont pas un centre générateur de subjectivité, et qui, à la différence du système verbal du DIL, ne se distinguent en rien sur le plan formel des expressions authentiquement anaphoriques.

Les emplois des localisateurs en DIL semblent cependant bien favoriser l'idée que le dispositif hybride, propre aux items à valeur transposée, se retrouve là encore. Un relevé à grande échelle (environ trois cents occurrences réparties sur les quatre siècles de la modernité), témoigne, par la variation observée, d'un libre choix à la production entre les expressions de forme déictique ou anaphorique :

Aussitôt l'homme s'embrouilla... Il ne se rappelait plus rien... Étaitelle venue hier?... On lui dirait ça au château... (Daudet, L'Évangéliste, 1883)

Des imprécations éclataient, il y eut une poussée pour se jeter sur le traître. Eh quoi! Il avait juré avec eux, la veille, et on le trouvait au 
fond, en compagnie des autres? C'était donc pour se foutre du monde ! (Zola, Germinal, 1885)

Les options prises dans les deux extraits sont divergentes, mais rien n'aurait empêché, sur le plan grammatical ou énonciatif, qu'elles fussent inverses: Etait-elle venue la veille? / Il avait juré avec eux, hier... ${ }^{11}$ Il apparaît donc que les localisateurs, à la différence des autres formes transposées, se prêtent en DIL à un jeu très libre de substitution qui se laisse interpréter comme le signe d'une parenté de fonctionnement.

Qu'une liberté de choix se présente ne signifie cependant pas que l'alternative soit dénuée d'enjeu. Selon le type de forme retenu, le dosage de la force expressive se trouve modulé, mais sans donner lieu à une opposition radicale entre subjectivité et objectivité puisque, telle est notre thèse, les formes de l'anaphore signifiant par transposition conservent un ancrage dans l'actualité du sujet représenté. L'examen de quelques cas appréhendés dans leur contexte doit permettre de vérifier cette vue :

Quand l'huissier se fut retiré à reculons, en saluant, il [Eugène Rougon] s'emporta, donna des coups de poing sur les meubles. On ne le laissait plus respirer ; la veille encore, on l'avait relancé jusque dans son cabinet de toilette, pendant qu'il se faisait la barbe. (Zola, Son Excellence Eugène Rougon, 1876)

Mme Hennebeau défaillait. Quelle abomination! Cette pauvre Cécile, si gaie ce jour-là, si vivante une heure plus tôt! Il fallut que Hennebeau fît entrer un instant sa femme dans la masure du vieux Mouque. (Zola, Germinal, 1885)

Les localisateurs la veille, ce jour-là, une heure plus tôt déploient en principe une référence temporelle qui se calcule relativement à un événement ou à un moment préalablement spécifié. Cette définition de base, correspondant à leur statut en langue, ne s'applique cependant pas aux emplois présentés ci-dessus qui, selon nous, s'inscrivent forcément dans la sphère de subjectivité construite par les marques énonciatives cooccurrentes. Dans le premier exemple, le localisateur intervient sous la portée d'un adverbe intensifiant (encore) qui scelle son appartenance à la conscience d'un sujet affecté. L'opération de subjectivation est ici due à un facteur extrinsèque à la donnée temporelle proprement dite et elle pourrait, en théorie, être le fait d'une évaluation narrative. Peut-on donc se prononcer sur le statut du localisateur lui-même ? Employée en fonction de complément de phrase, l'expression temporelle établit le cadre dans lequel les propos du 
protagoniste trouvent leur validité. À cette donnée pragmatique, on peut ajouter que le rattachement du localisateur à l'énonciation du personnage apparaît obligatoire pour une raison de cohérence aspectuelle : les procès ponctuels avait relancé/se faisait doivent impérativement être situés temporellement, afin que soit assuré un décrochement par rapport au régime duratif / itératif qui précède (on ne le laissait plus respirer). C'est d'ailleurs pourquoi le même énoncé, produit sans précision de temps, apparaîtrait difficilement acceptable. Relater les faits exaspérants et les situer forment donc une seule énonciation. De même que les deux faits singulatifs (relancer et faire la barbe), censément prononcés par le personnage et ressentis par lui comme un comble, sont livrés au titre d'explication de son propre comportement (il s'emporta, donna des coups de poing sur les meubles). Le geste de colère et la raison qui en est avancée procèdent donc d'un même mouvement de conscience, celui du personnage qui s'éprouve dans l'action. C'est à nouveau, comme on l'a vu s'exercer à propos du IL subjectivé, le principe du poids causal qui vérifie ici l'intégration des composantes et circonstances de l'énoncé à une conscience unique.

Dans le second exemple, l'intégration des indications temporelles à l'énonciation présumée du personnage advient par un autre biais. Leur rôle consiste à restreindre le domaine d'actualisation des attributs (si gaie, si vivante), directement associés, dans la syntaxe nominale, à la désignation démonstrative porteuse d'un point de vue. Tout se tient par conséquent dans une composition oralisante qui exprime la stupéfaction face à la soudaineté du trépas.

Jusqu'ici, nous avons montré le statut particulier des anaphoriques transpositeurs en les reliant à la subjectivité de l'énoncé. Leur valeur peut être encore mieux mise en évidence dans le cas où ils fonctionnent solidairement avec des déictiques :

La princesse nous apprit que le jeune violoniste était retrouvé. Il avait gardé le lit la veille à cause d'une migraine, mais viendrait ce soir et amènerait un vieil ami de son père qu'il avait retrouvé à Doncières. (Proust, Sodome et Gomorrhe, 1922)

Eh bien, c'était clair. La veille, quand elle était venue, il était déjà mort. On ne l'avait mis dans la petite chambre que pour cacher ça... on avait menti, hier, ce matin... on l'avait laissée dans l'ignorance, exprès... et pendant ce temps-là, Justin était seul, qui refroidissait avec les mouches, les affreuses mouches. (Aragon, Les Beaux quartiers, 1936) 
Dans le premier extrait, la configuration temporelle de la séquence en DIL donne à saisir la figure du violoniste selon deux perspectives inverses, l'une portée vers l'antériorité $(\mathrm{PqP}+$ la veille $)$, l'autre vers la postériorité (COND + ce soir). Plutôt qu'une succession chronologique, c'est en fait une opposition d'états, articulée par un mais contrastif, que le dispositif sert à décrire. Par leur orientation sémantique divergente, les propositions coordonnées expriment, dans l'ordre, une péripétie mondaine ainsi que l'issue heureuse qui est censée advenir. Mais la consécution événementielle ne relève pas ici d'un aménagement purement logique, correspondant à une stricte donnée de faits. On entend la réponse futile qui est apportée aux préoccupations de cette petite société. Et c'est bien l'annonce de la princesse, son actualité verbale, qui constitue le point de bascule assurant la transition entre un avant et un après. Cet ancrage se manifeste d'ailleurs par l'embrayeur temporel ce soir, qui trouve son origine dans le nunc de la locutrice $^{12}$. Quant au premier localisateur (la veille), il obéit à un fonctionnement similaire, et cela malgré sa forme anaphorique. La relation de distinction qui se tisse entre les deux indications de temps empêche en effet de les isoler en les répartissant sur deux plans énonciatifs différents. Elles se répondent, font système et construisent ensemble la pertinence du discours.

De façon plus décisive encore, le second exemple permet de comprendre l'absurdité qu'il y aurait à priver le localisateur la veille d'un ancrage dans la conscience du personnage. Parmi les expressions temporelles que contient l'énoncé, la veille et hier entretiennent un rapport de coréférence. Dans le principe, rien ne s'oppose à ce qu'un même moment soit désigné, selon leur modalité respective, par le récit et par un personnage. Mais dans le contexte présent, le recours à une forme anaphorique n'est pas destiné à fournir un cadre de vérité objectif aux événements. L'expression retenue, qui pourrait parfaitement être remplacée par son correspondant déictique hier, a pour intérêt de ménager une entrée progressive dans la conscience du personnage. Quoi qu'il en soit, c'est bien ce dernier qui recompose le déroulement des événements au moment où se découvre la tromperie.

Notre analyse des localisateurs s'en est jusqu'ici tenue au cas des indicateurs temporels. C'est qu'il est difficile de mettre en évidence une valeur transposée dans le choix des indicateurs spatiaux, car, du fait des paradigmes lexicaux disponibles, il n'y a souvent pas de différence formelle entre localisateurs anaphoriques et déictiques : là, à cet endroit peuvent fonctionner aussi bien par référence à un lieu donné, que par rapport à la place occupée par celui qui parle. Tout est donc affaire d'interprétation en contexte. Cependant, le DIL ne semble pas avoir l'usage de localisateurs spatiaux interprétables par transposition; il comprend : 
- soit de véritables anaphoriques relayant des données internes au discours du personnage, comme dans cet exemple où Virginie fait état de son passé à Gervaise :

Elle, pendant cinq ans, avait demeuré de l'autre côté de l'eau, au Groscaillou. C'était là qu'elle avait connu son mari, quand il était au service. Mais elle s'ennuyait, elle rêvait de revenir dans le quartier de la Goutte-d'or, où elle connaissait tout le monde. (Zola, L'Assommoir, 1877)

- soit de purs déictiques appartenant à l'énonciation du sujet de conscience. Ainsi l'adverbe là, même quand le DIL est précédé d'une séquence descriptive fixant le lieu de l'action, va fonctionner indexicalement, par ancrage dans la situation de parole ou de pensée représentée, et non par renvoi à l'indication de lieu préalablement fournie par le texte :

Dans un silence de désert, on l'entendait [la neige] fondre, ruisseler contre les murs, le long des vitres, s'égoutter dans les combles du toit, et, par moments, sur le feu de coke de la cheminée qu'elle éclaboussait.

Où était-il [Jean]? Que faisait-il là ? Peu à peu, dans la réverbération du petit jardin, la chambre lui apparaissait toute blanche, éclairée d'en bas, le grand portrait de Fanny dressé en face de lui, et le souvenir lui revenait de sa chute, sans le moindre étonnement. (Daudet, Sapho, 1884)

Quoique la chambre où le personnage reprend conscience ait déjà été mentionnée dans le récit, c'est à l'environnement perçu que réfère directement l'adverbe là du discours représenté ; en l'occurrence, la première interrogation (où était-il ?) dit assez que, dans l'expérience actuelle du sujet, les références spatiales antérieures n'ont provisoirement plus cours. Mais la rupture du système référentiel se produit aussi bien lorsque l'indicateur suit immédiatement une première localisation :

D'un geste, il [Madinier] commanda une halte, au milieu du salon carré. Il n'y avait là que des chefs-d'œuvre, murmurait-il à demi-voix, comme dans une église. (Zola, L'Assommoir, 1877)

À la lecture, là paraît d'abord reprendre anaphoriquement le lieu précédemment désigné. Mais la recatégorisation de l'énoncé en DIL imposée par le verbe attributif (murmurait-il) fait que l'adverbe doit s'entendre déictiquement. 


\section{Conclusion : la question des « voix » dans le DIL}

Nos analyses sont de nature à remettre en cause la description traditionnelle du DIL selon laquelle celui-ci mêlerait deux voix : celle du personnage représenté et celle du narrateur rapporteur. Cette conception est par exemple évoquée (et peut-être assumée) dans la citation suivante :

Dans le prolongement des perspectives de $\mathrm{M}$. Bakhtine on a peu à peu réalisé que dans [le DIL] on n'était pas confronté à une véritable énonciation mais qu'on entendait deux «voix» inextricablement mêlées, celle du narrateur et celle du personnage. (Maingueneau $2000: 105)$

Notons les guillemets qui appellent à concevoir la voix avec prudence, car on l'assimile parfois à l'énoncé matériel que profère un locuteur / scripteur ([18] Rabatel 2012 : 24), mais il lui arrive aussi d'être associée à la représentation sémantique d'un point de vue irréductible aux mots qui l'expriment ([19] Ducrot 1984 : 204). Les acceptions varient donc, et pourtant, dans l'histoire des théories du DIL, et notamment sous l'impulsion décisive de Volochinov (Bakthine), les voix sont littéralement audibles, parce qu'éminemment intonatives. Si deux voix se mêlent dans un même énoncé, c'est, dit-on, que deux locuteurs parlent ensemble. À l'encontre d'une telle conception, nous avons montré que les personnes, les temps verbaux et les localisateurs qui sont habituellement rapportés au système narratif sont en fait des quasi-indexicaux assumant la valeur définie par l'ancrage énonciatif que requiert l'interprétation par transposition. Dans ce type d'énoncé, IL est un SOI et non pas une troisième personne délocutée, l'IMP est un MAINTENANT et non pas un passé. De sorte qu'il n'y a pas lieu d'envisager l'implication d'une énonciation narrative; la parole du personnage est seulement modelée par un cotexte qui impose son apparence formelle au discours. Le résultat est une forme énonciative paradoxale qui n'est réductible ni à l'énonciation discursive, ni à l'énonciation historique. Ce n'est pas dire qu'il faille l'envisager comme un "mélange » de ces deux régimes, dont les unités hétérogènes seraient distribuées ou combinées syntagmatiquement dans la suite verbale; il s'agit plus fondamentalement d'une conversion des unités morphématiques elles-mêmes.

Une telle conception pourrait sembler problématique dans les cas de DIL en contexte de récit à la première personne où l'existence d'un narrateur est indéniable : 
Je regagnai la route et me mis à marcher, en la regardant, elle [en photographie]! Quelle joie qu'elle fût libre, qu'elle se fût sauvée! Certes, j'allais la rencontrer aujourd'hui ou demain, cette semaine ou la suivante, puisqu'elle l'avait quitté ! Elle l'avait quitté parce que mon heure était venue!

Elle était libre, quelque part dans le monde! (Maupassant, « À vendre », 1885)

Mais le DIL se comprend ici encore selon un même mouvement de transposition: JE, en tant que forme adaptée à l'organisation narrative, désigne le sujet de conscience et non pas le rapporteur du discours. Le fait qu'il puisse y avoir parfois, dans les récits homodiégétiques, équivoque entre les énoncés attribuables au JE-narrateur et ceux qui représentent le discours du JE-personnage concerne l'interprétation elle-même et ne signifie pas l'indistinction des structures énonciatives :

Il nous faut donc identifier parmi les phrases du récit à la première personne et au passé deux types de phrases qui s'excluent mutuellement, même si tel énoncé particulier peut présenter quelque ambiguïté entre les deux types. Dans un cas le je se borne à raconter les événements passés et dans l'autre il en fait l'expérience vécue. ([5] Banfield, 1995 : 246)

\section{Références}

1. J. Authier-RevuZ, «Repères dans le champ du discours rapporté », L'Information grammaticale, 55 \& 56, Louvain, pp. 38-42 \& 10-15, (1992 \& 1993).

2. J. AuthiER-RevuZ, «La représentation du discours autre: un champ multiplement hétérogène ", in Le Discours rapporté dans tous ses états, J. M. Lopez Muñoz, S. Marnette \& L. Rosier (dirs.), Paris, L'Harmattan, pp. 35-53, (2004).

15. Ch. BAlly, "Figures de pensée et formes linguistiques I», GermanischRomanische Monatsschrift, VI/7, Heidelberg, pp. 405-422, (1914).

4. A. BANFIELD, «Le style narratif et la grammaire des discours direct et indirect », trad. franç. Change, 16/17 (« La critique générative »), Paris, Seuil, (1973).

5. A. BANFIEld, Phrases sans parole, trad. franç., Paris, Seuil, $\left(1995,{ }^{1} 1982\right)$.

3. A.-M. BerthonNeAu \& G. KLeIBER, «Subordination et temps grammaticaux : l'imparfait en discours indirect », Le Français moderne, LXV/2, Paris, pp. 113141, (1997).

6. H. CURAT, Morphologie verbale et référence temporelle en français moderne, Genève, Droz, (1991).

19. O. Ducrot, Le Dire et le dit, Paris, Minuit, (1984). 
9. J.-D. Gollut \& J. ZuFFEREY, « La désignation de l'énonciateur dans le discours indirect libre ", Marges et contraintes du discours indirect libre, Fabula colloques en ligne (http://www.fabula.org/colloques/document3361.php), (2016).

16. L. Gosselin, Sémantique de la temporalité. Un modèle calculatoire et cognitif du temps et de l'aspect, Louvain-La-Neuve, Duculot, (1986).

10. G. KleIBER, Anaphores et pronoms, Louvain-la-Neuve, Duculot, (1994).

17. D. Maingueneau, Éléments de linguistique pour le texte littéraire, Paris, Nathan Université, (2000).

8. R. PASCAL, The Dual Voice: Free Indirect Speech and Its Functioning in the XIX ${ }^{\text {th }}$ Century European Novel, Manchester University Press, (1977).

11. J. Perry, "The Problem of the Essential Indexical», in The Problem of the Essential Indexical and other Essays, Oxford, Oxford University Press, pp. 32-52, (1993).

13. C.M. POPESCU, «Conditionnel ou futur dans le passé ? L'histoire d'un marqueur de relation anaphorique ", Zeitschrift für französische Sprache und Literatur, 123/1, Stuttgart, pp. 12-32, (2013).

18. A. RABATEL, "Positions, positionnements et postures de l'énonciateur", TRANEL, 56, Neuchâtel, pp. 23-42, (2012).

12. A. Reboul, «Communication, fiction et expression de la subjectivité », Langue française, 128, Paris, pp. 9-29, (2000).

14. A. TOBLER, "Vermischte Beiträge zur französischen Grammatik », Zeitschrift für Romanische Philologie, XI, Halle, pp. 431-461, (1887).

7. V.N. VolochinOV, "Le discours indirect libre en français, en allemand et en russe ", Le Marxisme et philosophie du langage. Les problèmes fondamentaux de la méthode sociologique dans les sciences du langage, trad. P. Sériot \& I. Tylkowski-Ageeva, Limoges, Lambert-Lucas, pp. 423- 465, (2010, $\left.{ }^{1} 1929\right)$.

\footnotetext{
${ }^{1}$ Nous remercions Gilles Philippe pour ses remarques et ses conseils.

2

2 [3] A.-M. Berthonneau et G. Kleiber (1997) ont pointé les limites de l'explication par pure dérivation formelle à partir d'un discours d'origine : la base de repérage temporelle parfois incertaine en contexte indirect libre, les énoncés indirects sans correspondants directs possibles, l'ambiguïté de l'imparfait susceptible de transposer un présent ou un imparfait, la non transposabilité du plus-que-parfait, la nécessité d'ajustements ad hoc telle que la non transposition au passé d'un verbe encore valide au moment de l'énonciation rapportante.

La grammaire générative a de son côté montré que, du fait des différences de leurs structures profondes, il n'est pas possible de dériver les uns des autres les discours direct, indirect et indirect libre (cf. [4, 5] Banfield, 1973 \& $1995\left({ }^{1} 1982\right)$ ).

${ }^{3}$ Nous avons montré ailleurs que les formes transposées désignant le SOI ne se prêtent pas, au contraire des pronoms anaphoriques, à une libre substitution par un nom propre ou une description définie (cf. [9] Gollut \& Zufferey 2016).

4 «Quelle grossière erreur d'être venu. Il aurait dû rester chez lui et lire son livre, pensa Peter Walsh » (trad. Pascale Michon, Le Livre de Poche, p. 189).
} 
5 Nous adoptons les abréviations suivantes: PR (présent), FUT (futur), IMP (imparfait), PS (passé simple), PqP (plus-que-parfait), COND (conditionnel).

${ }^{6}$ Voir par ex. [5] Banfield (1995: 324), [12] Reboul (2000:22).

7

7 Les linguistes ne sont pas unanimes sur la possibilité d'occurrence d'un PS en contexte indirect libre. Si Ducrot en admet l'éventualité, Guillaume ou Weinrich l'excluent tout à fait. Pour notre part, nous dirons simplement que le PS n'est pas de nature à pouvoir fonctionner comme un temps transpositeur et reste donc en marge du processus de subjectivation dont nous voulons rendre compte.

${ }^{8}$ Dans une optique cognitive, Gosselin ([16] 1996: 86) pose le principe d'une « contrainte aspectuelle sur la simultanéité »: «[...] un sujet ne peut embrasser du regard un procès qui est simultané à sa perception. De façon plus technique, cela revient à dire que les bornes du procès ne sont pas accessibles à partir de l'intervalle de référence lorsque celui-ci coïncide avec l'intervalle de l'énonciation. »

9 «[...] si la subordination temporelle était un phénomène d'une certaine généralité, elle pourrait (ou devrait ?) s'exercer également sur des situations déjà à l'imparfait. » (Berthonneau \& Kleiber 1997 : 130)

10

On se gardera de confondre les expressions proprement anaphoriques et celles qui en ont l'aspect tout en admettant un sens par transposition : «Eh bien, c'était clair. $L a$ veille, quand elle était venue, il était déjà mort. On ne l'avait mis dans la petite chambre que pour cacher ça... on avait menti, hier, ce matin... on l'avait laissée dans l'ignorance, exprès... et pendant ce temps-là, Justin était seul, qui refroidissait avec les mouches, les affreuses mouches» (Aragon, Les Beaux quartiers, 1936). Seule la première des deux formes soulignées intègre notre problématique.

${ }^{11}$ Des conditions d'emploi contraignantes peuvent advenir lorsqu'un premier renvoi temporel est effectué à un moment antérieur ou postérieur à l'action racontée. Afin d'éviter un repérage indu par rapport au moment énoncé et donc l'engagement d'un calcul référentiel erroné, le recours à un localisateur déictique s'impose. Par exemple : « Des femmes passèrent dans la cour avec un bard d'où dégouttelait du linge. En les apercevant par les carreaux, elle se rappela sa lessive ; l'ayant coulée la veille, il fallait aujourd'hui la rincer; et elle sortit de l'appartement» (Flaubert, Un Cour simple, 1877). «Il fallait la rincer ce jour-là » ne conviendrait pas en raison de l'ambiguité qui en résulterait.

${ }_{12}$ Bien que Proust neutralise parfois l'opposition ce soir / ce soir-là, le cas présent relève forcément du régime déictique en vertu de la postériorité signifiée par le temps verbal (viendrait). 